\title{
ASSESSMENT OF HEMODYNAMIC PARAMETERS DISTRIBUTION IN NORMAL CAROTID ARTERY BY USING COMPUTATIONAL FLUID DYNAMICS BASED ON CE-MRA DATA
}

\author{
ASSESSMENT OF HEMODYNAMIC PARAMETERS DISTRIBUTION IN NORMAL \\ CAROTID ARTERY BY USING COMPUTATIONAL FLUID DYNAMICS BASED ON \\ CE-MRA DATA
}

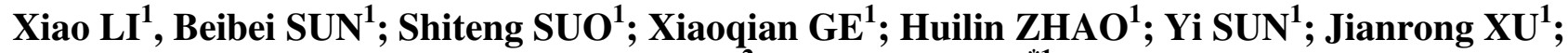 \\ Bulang $\mathrm{GAO}^{2}$; Xiaosheng $\mathrm{LIU}^{* 1}$ \\ 1. Department of Radiology, Renji Hospital, School of Medicine, Shanghai Jiaotong University, Shanghai 200127, China; 2. \\ Department of Medical Research, Shijiazhuang First Hospital, Hebei Medical University, Shijiazhuang 050011, China. \\ Co-first author statement: Dr. Xiao Li and Dr. Beibei Sun, contributed equally to this work and are considered to be co-first \\ authors.*Author for correspondence: miaxiaosheng@gmail.com
}

\begin{abstract}
This study was to analyze the in vivo distribution of wall shear stress (WSS), velocity and inplane pressure difference in normal carotid artery by using computational fluid dynamics (CFD) based on contrastenhanced MRA (CE-MRA) data and to determine whether there were differences in these hemodynamic parameters between atherosclerosis-free and atherosclerosis-prone areas. CE-MRA was performed on 16 normal carotid arteries to obtain carotid three-dimensional surface data. CFD analysis was then performed to estimate those parameters in atherosclerosis-free (distal common carotid artery and distal internal carotid artery) and atherosclerosis-prone (carotid bifurcation) areas. One-way analysis of variance (ANOVA) was conducted to analyze differences among these three areas. CFD analysis revealed that WSS and velocity were significantly lower in carotid bifurcation compared to distal common carotid artery (CCA) $(P=0.011, P<0.001$, respectively) and distal internal carotid artery (ICA) $(P<0.001, P<0.001$, respectively). While in-plane pressure difference was significantly higher in carotid bifurcation compared to distal CCA $(P$ $<0.001)$ and distal ICA $(P=0.005)$. Hemodynamic environment in carotid bifurcation of normal carotid artery which assessed by using CFD analysis appeared to be with lower WSS and lower velocity, while with higher in-plane pressure difference. These characterizations might facilitate the initiation of atherosclerosis.
\end{abstract}

KEYWORDS: Wall shear stress. Velocity. In-plane pressure difference. Computational fluid dynamics. Carotid atherosclerosis

\section{INTRODUCTION}

Carotid atherosclerosis is responsible for the majority of the stroke, which increased by $>100 \%$ in low- and middle-income countries over the past four decades (MESCHIA; BUSHNELL et al., 2014). Atherosclerosis is a systemic disease characterized with intimal lesion formation in the artery. It is prone to initiate at the bend or major branches of arterial network, such as the bifurcation of carotid artery (BIJARI; WASSERMAN et al., 2014). Systemic cardiovascular risk factors can't explain the focal characters. Studies on carotid bifurcation geometry provide sufficient evidence that hemodynamic factors are associated with the local onset and progression of the atherosclerotic disease (CHEN; CANTON et al., 2015; ZHOU; MENG et al., 2017). Among the various hemodynamic factors, wall shear stress (WSS) is one of the major contributors to the development of carotid atherosclerosis. The mechanisms that WSS alters endothelial phenotype and function at the genetic, molecular and cellular level, consequently promoting the formation of atherosclerotic plaque have been elucidated (MALEK; ALPER et al., 1999; LEHOUX; JONES, 2015). Although WSS analysis has been applied to carotid bifurcation, more factors like in-plane pressure difference were scarce.

Our study aims to investigate the distribution of WSS, velocity and in-plane pressure difference in the atherosclerosis-free and atherosclerosis-prone areas of normal carotid artery. This would allow to elaborate the hemodynamic factors influencing the initiation of atherosclerosis.

\section{MATERIAL AND METHODS}

\section{Patients}

Sixty-five persons were scanned on a 3.0T MR scanner (Achieva; Philips Healthcare, Best, Netherlands) with contrast-enhanced magnetic resonance angiography (CE-MRA) imaging protocol in department of radiology, RenJi hospital 
from September 2012 to September 2015. Patients who met the following inclusion criteria were enrolled into our study: (1)over 40 years old, (2)systemic vascular diseases like vasculitis were not find clinically, (3)normal carotid artery with ICA wall thickness $<1.38 \mathrm{~mm}$ and CCA wall thickness $<2.06 \mathrm{~mm}$ which measured in MR black blood sequence (ASTOR; SHARRETT et al., 2010). Finally, 12 patients with 16 normal carotid arteries were enrolled in the study, which including 58\% male, $50 \%$ smoke, $37.5 \%$ drink, $37.5 \%$ diabetes, $50 \%$ hypertension and $33.3 \%$ hyperlipidemia. The study protocol was approved by the Institutional Review Board of Renji Hospital. Informed consent was obtained from all of the subjects.

\section{D geometric reconstructions}

3D angiography obtained from CE-MRA were reconstructed and rendered by using Amira software (Amira 4.1.2 Visage Imaging, San Diego, CA). Branches of external carotid artery (ECA) were virtually removed for their small size. Smoothing surface and polishing gaps of the 3D reconstructed datasets were performed using Meshlab software (Meshlab v1.2.2, Visual Computing Lab, ISTI, CNR). High-resolution polyhedral meshes were generated with Harpoon (4.3 SHARC Ltd., Manchester, UK) at a count of approximate 400000 cells. Blood was modeled with a density of $1060 \mathrm{~kg} / \mathrm{m}^{3}$ as a nonNewtonian fluid to better simulate the known shear-rate dependence of its viscosity. Steady-state simulations for the model validation assuming nonNewtonian behavior of blood were performed using a viscosity value of 3.5 $\mathrm{cP}$. The vessel wall was assumed to be rigid, and velocity conditions obeyed nonslip and nonpenetration constraints at the wall (GAO; BAHAROGLU et al., 2013). Artery geometry and hemodynamic parameters profiles were depicted and exported by Ensight software (Version9; CEI, Apex, NC).

\section{Data analysis}

The computational fluid dynamics (CFD) simulation was performed from common carotid artery (CCA) to internal carotid artery (ICA) and ECA. The outlet and inlet were set perpendicular to the centerline for carotid artery models. The inlet surface was $5 \mathrm{~cm}$ away from the bifurcation to minimize the computational error caused by the boundary condition (SCHIRMER; MALEK, 2007). Analyses on 19 cross-sectional planes were performed to gain the data of WSS and pressure. All planes were perpendicular to the centerline of the artery. To ensure the consistent definition of 19 analysis planes for all subjects, we made the plane 9 at ICA and transit the apex of bifurcation as reference. The other planes were generated by shifting the plane center upstream or downstream at $2 \mathrm{~mm}$ intervals. The locations of the artery walls were defined by automatically outlining contours on the measured planes. The wall hemodynamic parameters of WSS and pressure were collected point-by-point on each contour. We defined WSSmin and Pressuremin as the minimum WSS and minimum Pressure at each plane, respectively. And Pressuremax was defined as the maximum pressure at each plane. In-plane pressure difference was defined as pressuremax minus pressuremin. Furthermore, CFD analysis was coupled with minor cut planes along the outer wall from CCA to ICA to enable analysis of the velocity profiles from all case-specific reconstructions. The minor cut planes set $0.5 \times 0.5 \mathrm{~mm}$ were placed in the coronal plane attached to each cross-sectional plane to obtain the velocity. Velocity in the minor plane was averaged to represent the velocity in the outer lumen corresponded with 19 cross-sectional planes. As a result, 19 cross-sectional planes and 19 minor planes were achieved. Because atherosclerotic lesions are typically located in bifurcation, especially the outer wall of proximal ICA (bulb), three regions of interest were defined: region 1-distal CCA contained with planes 1 to 4 ; region 2-bifurcation contained with planes 8 to 11 ; region 3-distal ICA contained with planes 16 to 19(ZARINS; GIDDENS et al., 1983). The 19 minor plates were sorted the same as the 19 cross-sectional planes. (Figure 1A)

\section{Statistical analysis}

Continuous variables were reported as mean \pm SD. Measure ANOVA was used to analyze between the atherosclerosis-free and atherosclerosisprone areas of WSS, velocity and in-plane pressure difference. A Bonferroni-corrected $P$ value of less than $0.05 / 3$ was considered to indicate significant in the case of the post-hoc Wilcoxon signed rank test (3 paired comparisons at 3 regions). All statistical analyses were completed with IBM SPSS Statistics 22.0 software (Armonk, NY).

\section{RESULTS AND DISCUSSION}

The study focused the detailed hemodynamic characteristics in normal carotid artery bifurcation. Consistent with previous studies, we found that potentially atherogenic parameters in normal carotid artery were predominantly concentrated at the outer wall of the physiologically 
dilated bulb located in bifurcation (LEE; ANTIGA et al., 2008).

WSS played a key role in the initiation and progression of the atherosclerosis for its interaction with vascular endothelial. Figure 1B summarized the distribution of WSS in the carotid artery and exhibited a visible decrease in the area of bifurcation. The curve (Figure 3A) indicated a trough of WSSmin in planes 8 to 11 and the values of WSSmin in the area tended to be 0 . The average WSSmin magnitude of 16 arteries in region 2 was $0.2664 \pm 0.1988 \mathrm{~Pa}$, both significantly lower than that in region $1(1.6072 \pm 0.7750 \mathrm{~Pa}, P=0.011)$ and region $3(7.8482 \pm 3.3075 \mathrm{~Pa}, \quad P<0.001)$ (Figure4A). Figure 2A showed the curve of WSS in plane 9 versus the circumferential distance and illustrated that the WSS minimum was located in the outer wall of plane 9. The same were WSS distribution in the other planes in region 2 . Atherosclerosis developed more readily in regions where the WSS was expected to be low, such as at the outer wall of proximal ICA. In the early stage of the hemodynamic investigation, they described the detailed flow patterns in various glass models and found WSS was lowest in carotid bulb (ZARINS; GIDDENS et al., 1983b; KU; GIDDENS et al., 1985). Gnasso et al (GNASSO A, 1997) made the same conclusion that WSS was lower in the carotid arteries where plaques were present than in plaquefree arteries. Our study focused on in-vivo normal carotid artery bifurcation by using MR and CFD was consistent with these previous studies.

The flow recirculation regions with low velocity could be seen clearly from the instantaneous velocity vector in coronal plane and cross-sectional plane 9 of the carotid artery colored by velocity, as illustrated in figure1D. Starting with laminar flow in the common carotid artery, the blood flow changed into a considerable turbulent and slow pattern in the proximal ICA for the bifurcation served as a flow divider and the expansion of bulb. Plane-by-plane analysis (Figure 3B) showed a smaller velocity at region 2 with the result of $0.12 \pm 0.12 \mathrm{~m} / \mathrm{s}$, versus the value of $0.84 \pm$ $0.31 \mathrm{~m} / \mathrm{s}$ in region $1(P<0.001)$ and $1.42 \pm 0.49 \mathrm{~m} / \mathrm{s}$ in region $3 \quad(P<0.001)$ (Figure 4B). Velocity decreased with flow separation phenomenon, which occurred when the vascular cross-sectional area expanded rapidly. This finding was consistent with the previous studies (MORBIDUCCI; PONZINI et al., 2011; PEDERSEN; PIHL et al., 2014). With low velocity accompanied with vortex, the elements in the flood like platelet and lipid were prone to separate out and induced the formation of atherosclerosis in the local wall (FAN; SAKAI et al., 2010).

In-plane pressure difference distributions along the planes were shown in Figure 3C. In the region 2 , the in-plane pressure difference abruptly increased to its peak value and sharply fell off in the distal ICA planes. The in-plane pressure difference in region 2 located in the bifurcation was the highest compared with the region 1 and region 3 (243 \pm 149 $\mathrm{Pa}$ vs $40 \pm 22 \mathrm{~Pa}, P<0.001 ; 243 \pm 149 \mathrm{~Pa}$ vs $170 \pm 48$ $\mathrm{Pa}, P=0.005$; respectively) (Figure $4 \mathrm{C}$ ). Figure $2 \mathrm{~B}$ showed the curve of pressure in plane 9 versus the circumferential distance and illustrated that pressure maximum was located in the inner wall of bifurcation, and pressure minimum was located in the outer wall of bifurcation. It illustrated that the in-plane pressure difference between innerwall and outwall in region 2 is the largest among the three regions. These findings were mirrored by the Figure1C, visualization of pressure characteristics of the carotid artery. Consequently, larger in-plane pressure difference was found in the carotid bifurcation compared with the other regions in the carotid artery. Li et al (LI; TAVIANI et al., 2009) studied pressure distribution on stenotic carotid artery and drew the conclusion that pressure may be the main mechanical trigger for plaque rupture. Sui et al (SUI; GAO et al., 2015) found significant differences in upstream to stenosis center of pressure, as well as in stenosis center to downstream. These studies discussed the distribution of pressure in stenotic carotid artery. And our study was the first one to discuss pressure distribution in normal carotid artery and try to find the association between pressure and initiation of plaque. We found that the in-plane pressure difference in the bifurcation was the largest among the three regions. The pressure difference might cause a water hammer effect, leading to aggregation of elements of flood to outer wall, followed by the atherosclerotic plaque. The results showed evidence that high in-plane pressure difference was associated with sites of plaque initiation. 


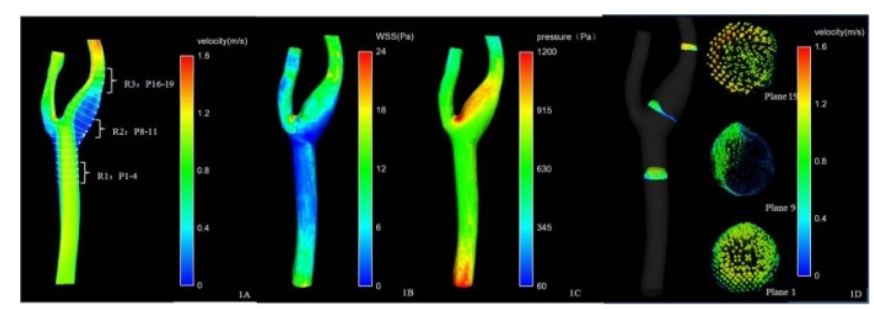

Figure 1. Analysis planes (A) and distribution of WSS (B), pressure (C) and velocity (D) in normal carotid artery.

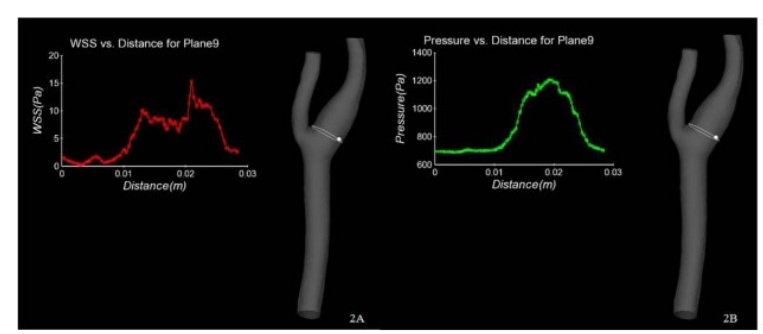

Figure 2. WSS (A) and pressure (B) in plane 9 versus circumferential distance.

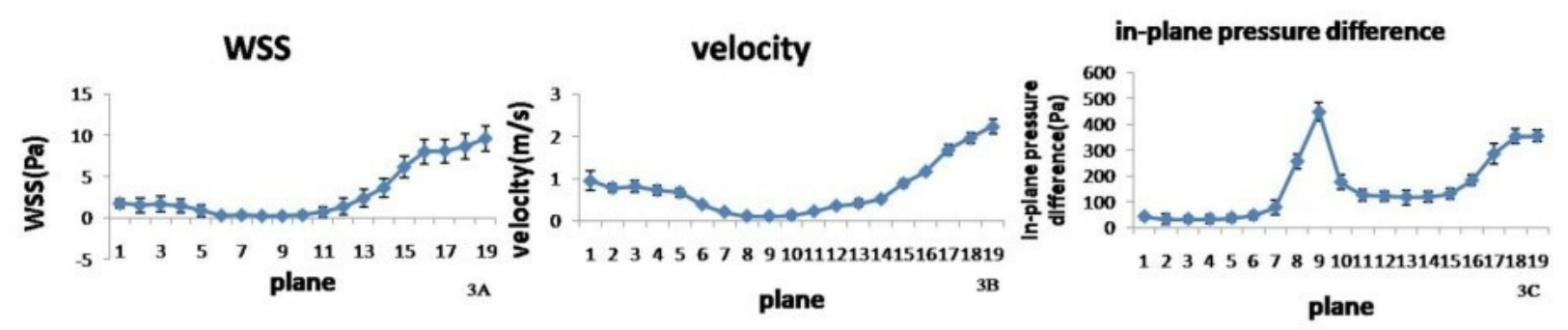

Figure 3. WSSmin (A), velocity (B) and in-plane pressure difference (C) in 16 normal carotid arteries versus planes.
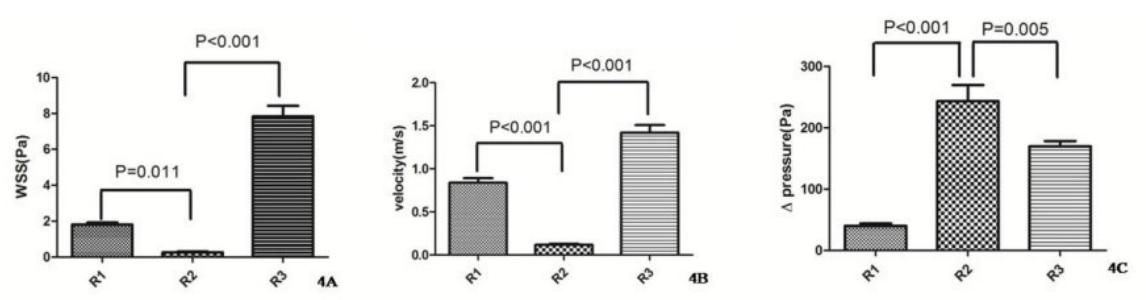

Figure 4. WSSmin (A), velocity (B) and in-plane pressure difference $(\mathrm{C})$ related to 3 regions. $\mathrm{R} 1$ : region1; R2: region2; R3: region3; $\triangle$ pressure: in-plane pressure difference

\section{CONCLUSION}

Results confirm that carotid bifurcation of normal carotid artery studied using CFD analysis appears to harbor a hemodynamic environment characterized with lower WSS and velocity while higher in-plane pressure difference, favors the initiation of atherosclerosis. These results could provide method and data to guide further experimental studies and understand the hemodynamic factors of the multifactorial driving forces behind the initiation and progression of the carotid atherosclerosis.

\section{ACKNOWLEDGEMENTS}

This work was supported by the National Science Funds of China (No. 81271575, 81401374, 81571630) and the SJTU Medical-Engineering Cross-Cutting Research Project (Grant no. YG2015MS53). 


\section{REFERENCES}

Astor B. C., Sharrett A. R., Coresh J., Chambless L. E., Wasserman B. A. Remodeling of carotid arteries detected with MR imaging: atherosclerosis risk in communities carotid MRI study. Radiology, v. 256, n. 3, 2010-09-01, p. 879-86. 2010.

Bijari P. B., Wasserman B. A, Steinman D. A. Wasserman. Carotid Bifurcation Geometry Is an Independent Predictor of Early Wall Thickening at the Carotid Bulb. Stroke, v. 45, n. 2, 2014-02-01, p. 473-478. 2014.

Chen Y. M., Canton G. K., William S., Chiu B. Modeling hemodynamic forces in carotid artery based on local geometric features. Medical \& Biological Engineering \& Computing, 2015-11-17. 2015.

Fan L., Sakai J., Bessho S., Wada S., Karino T. Effect of a disturbed flow on adhesion of monocytes to a model of an arterial wall. Biorheology, v. 47, n. 1, 2010-01-20, p. 15-29. 2010.

Gao B., Baharoglu M. I., Cohen A. D., Malek A. M. Y-stent coiling of basilar bifurcation aneurysms induces a dynamic angular vascular remodeling with alteration of the apical wall shear stress pattern. Neurosurgery, $v$. 72, n. 4, 2013-04-01, p. 617-29. 2013.

Gnasso A., Irace C., Carallo C. In Vivo Association Between Low Wall Shear Stress and Plaque in Subjects With Asymmetrical Carotid Atherosclerosis. Stroke, v. 28, n. 5, 1997-05-01, p. 993-8. 1997.

Ku D. N., Giddens D. P., Zarins C. K., Glagov S. Pulsatile flow and atherosclerosis in the human carotid bifurcation. Positive correlation between plaque location and low oscillating shear stress. Arteriosclerosis, v. 5, n. 3, 1985-05-01, p. 293-302. 1985.

Lee S. W., Antiga L., Spence J. D., Steinman D. A. Geometry of the Carotid Bifurcation Predicts Its Exposure to Disturbed Flow. Stroke, v. 39, n. 8, 2008-08-01, p. 2341-2347. 2008.

Lehoux, S. e E. A. Jones. Shear stress, arterial identity and atherosclerosis. Thromb Haemost, v. 115, n.4, 2015-12-17. 2015.

Li Z. Y., Taviani V., Tang T., Sadat U., Young V., Patterson A., Graves M., Gillard J. H. The mechanical triggers of plaque rupture: shear stress vs pressure gradient. Br J Radiol, v. 82 Spec No 1, 2009-01-01, p. S3945. 2009.

Malek A. M., Alper S. L., Izumo S. Hemodynamic shear stress and its role in atherosclerosis. JAMA, v. 282, n. 21, 1999-12-01, p. 2035-42. 1999.

Meschia J. F., Bushnell C., Boden-Albala B., Braun L. T., Bravata D. M., Chaturvedi S., Creager M. A., Eckel R. H., Elkind M. S. V., Fornage M., Goldstein L. B., Greenberg S. M., Horvath S. E., Iadecola C., Jauch E. C., Moore W. S., Wilson J. A. Guidelines for the Primary Prevention of Stroke. Stroke, v. 45, n. 12, p. 3754-3832. 2014. https://doi.org/10.1161/STR.0000000000000046

Morbiducci U., Ponzini R., Rizzo G., Cadioli M., Esposito A., Montevecchi F. M., Redaelli A. Mechanistic insight into the physiological relevance of helical blood flow in the human aorta: an in vivo study.

Biomechanics and Modeling in Mechanobiology, v. 10, n. 3, p. 339-355. 2011.

https://doi.org/10.1007/s10237-010-0238-2

Pedersen M. M., Pihl M. J., Haugaard P., Hansen K. L., Lange T., Lonn L., Nielsen M. B., Jensen J. A. Novel flow quantification of the carotid bulb and the common carotid artery with vector flow ultrasound. Ultrasound Med Biol, v. 40, n. 11, 2014-11-01, p. 2700-6. 2014.

Schirmer C. M., Malek A. M. Prediction of complex flow patterns in intracranial atherosclerotic disease using computational fluid dynamics. Neurosurgery, v. 61, n. 4, 2007-10-01, p. 842-51. 2007. 
Sui B., Gao P., Lin Y., Jing L., Sun S., Qin H. Hemodynamic parameters distribution of upstream, stenosis center, and downstream sides of plaques in carotid artery with different stenosis: a MRI and CFD study. Acta Radiologica, v. 56, n. 3, 2015-03-01, p. 347-354. 2015.

Zarins C. K., Giddens D. P., Bharadvaj B. K., Sottiurai V. S., Mabon R. F., Glagov S. Carotid bifurcation atherosclerosis. Quantitative correlation of plaque localization with flow velocity profiles and wall shear stress. Circ Res, v. 53, n. 4, 1983-10-01, p. 502-14. 1983.

Zhou H., Meng L., Zhou W., Xin L., Xia X., Li S., Zheng H. R., Niu L. L. Computational and experimental assessment of influences of hemodynamic shear stress on carotid plaque. BioMedical Engineering OnLine, $v$. 16, n. 1. 2017. https://doi.org/10.1186/s12938-017-0386-z 